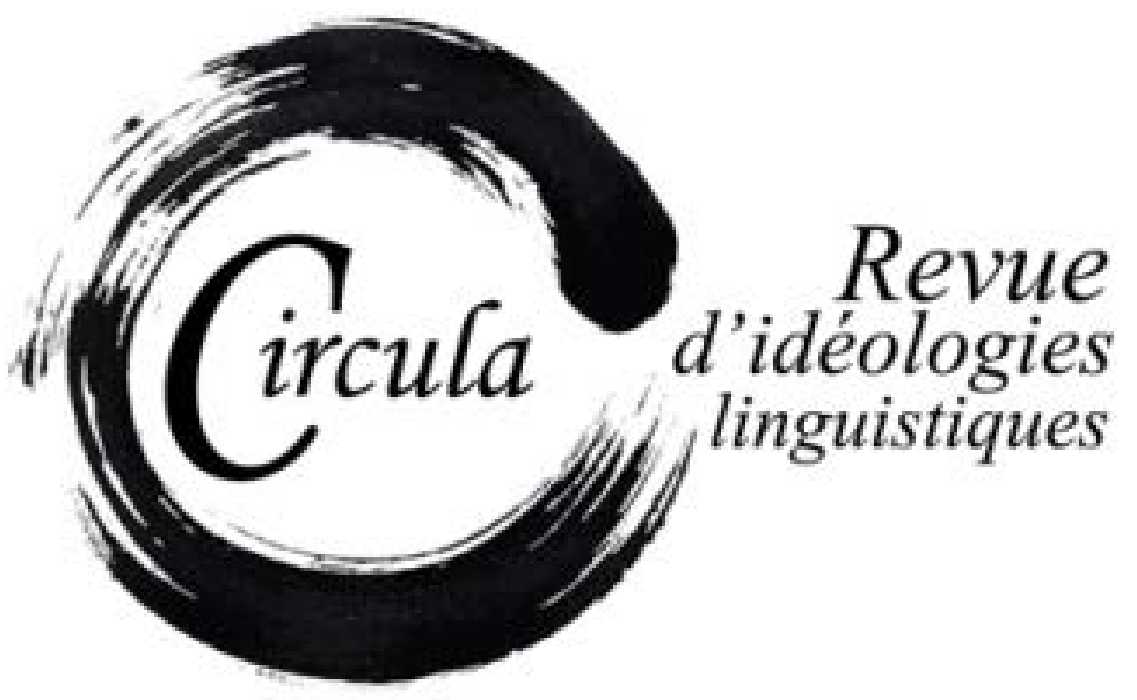

TITRE: REPRÉSENTATIONS DES LANGUES ET DES IDENTITÉS : LE CAS DE LA DIASPORA SERBE AU SUD DE LA FRANCE

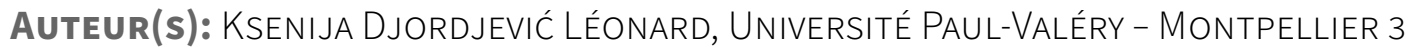

Revue: CirCula, NUMÉRO 3

PAGES: $43-61$

ISSN: 2369-6761

DiRecteurs: Bruno MAURER, UniVERSité PAUl-VALÉRY - MONTPELLIER 3

URI: HTTP://HDL.HANDLE.NET/11143/9695

DOI: $10.17118 / 11143 / 9695$ 


\section{Représentations des langues et des identités : le cas de la diaspora serbe au sud de la France}

Ksenija Djordjević Léonard, Université Paul-Valéry - Montpellier 3 ksenija.leonard@univ-montp3.fr

Résumé : Nous proposons d'interroger, dans cette contribution, les représentations sociolinguistiques des immigrés issus de la diaspora ex-yougoslave, installés au sud de la France, qui vivent au quotidien le contact entre la langue maternelle (langue de la maison ou de l'environnement familial) et le français (langue de l'environnement social). Les réflexions que nous proposons ici s'insèrent dans le projet « Représentations des langues et des identités en Méditerranée en contexte plurilingue » (EA 739 Dipralang). Notre étude de cas permet d'explorer les frontières de ce projet (langues de migration plutôt que langues minoritaires d'implantation historique) et le rôle des représentations sociolinguistiques dans la structuration des identités de leurs locuteurs.

Mots-clés : représentations des langues; identités ; France ; diaspora serbe ; langue serbe ; langue française

Abstract: This article deals with the following research question: how do migrants from the Yugoslavian diaspora settled in Southern France and constantly involved in dual communication (use of Serbian and French at home, use of French only in social settings) convey their sociolinguistic attitudes? It is part of a broader project, "Language and Identity in the Mediterranean in multilingual settings" (EA 739 Dipralang). Our case study addresses crucial issues from this project on minority languages that resort to migration rather than to historical heritage. We also explore to what extent sociolinguistic attitudes interfere with the shaping of migrant identity, in Southern Europe.

Keywords: representations of languages ; identities ; France ; Serbian diaspora ; Serbian language ; French language 


\section{Introduction}

Nous proposons d'interroger, dans cette contribution, les représentations sociolinguistiques des immigrés issus de la diaspora ex-yougoslave, installés au sud de la France, notamment dans l'Hérault et les Bouches-du-Rhône. Nous nous concentrerons sur les locuteurs du serbe, installés dans ces deux départements, qui vivent au quotidien le contact entre - au minimum - deux langues: la langue maternelle (langue de la maison ou de l'environnement familial) et le français, langue de l'environnement social.

Notre corpus sera composé d'éléments de discours recueillis auprès de trois générations d'ex-Yougoslaves: ceux installés dans la région depuis plusieurs décennies, ceux arrivés après l'éclatement du pays, et ceux qui sont nés en France ${ }^{1}$, de parents serbes. Même si ces trois catégories de locuteurs se distinguent souvent par la compétence linguistique (aussi bien en langue maternelle qu'en français), par la relation aux langues constitutives de leur répertoire linguistique et par le rôle que jouent ces langues dans leur construction identitaire, dans cette étape du travail nous les réunirons dans un seul et même corpus : les immigrés serbes (1e et 2e génération), de langue maternelle serbe, ayant une compétence satisfaisante ou très satisfaisante (locuteurs bilingues) en français, langue du pays d'accueil.

Les réflexions que nous proposons ici s'insèrent dans le projet « Représentations des langues et des identités en Méditerranée en contexte plurilingue ", développé à l'Université de Montpellier 3, par Bruno Maurer, en 2013. Ce projet, s'insérant dans l'axe "Construction des altérités culturelles et des sociétés plurielles en Méditerranée ", qui a bénéficié du soutien de la MSH de l'Université de Montpellier 3, s'est proposé d'explorer, durant deux années (2013-2014), les représentations des langues et des identités dans un certain nombre de territoires méditerranéens (France, Italie, Algérie, Croatie), à travers le rapport dominant/dominé, ou majorité/minorité. Notre étude de cas permet d'explorer les frontières de ce projet (langues de migration plutôt que langues minoritaires d'implantation historique) et le rôle des représentations sociolinguistiques dans la structuration des identités de leurs locuteurs. On sort ici du cadre d'une diglossie classique pour intégrer de multiples dimensions qui structurent la biographie langagière et l'identité du locuteur, aussi bien historiques que sociales ou psychologiques.

\section{Approche théorique des représentations sociolinguistiques}

La principale notion mobilisée dans cette étude de cas est la notion de représentation. Pour définir ce concept, il faut prendre en compte les apports de toutes les disciplines qui se le sont approprié. On pourrait rappeler ici aussi bien la réflexion sur le plan philosophique menée par E. Kant, que l'op-

1. Nous nous éloignons ici délibérément de la définition classique d'immigré (dans le contexte français : personne née à l'étranger, mais habitant en France). 
position entre les représentations individuelles et collectives du sociologue E. Durkheim, ainsi que l'apport que constituent les travaux du psychologue S. Moscovici, qui a eu le mérite de mobiliser tout un courant de recherche sur les représentations - en ce qui le concerne, essentiellement sociales, qui a dépassé, depuis, les limites de sa discipline. De nos jours, la notion de représentation intéresse et interpelle la plupart des sciences humaines, au point que certains pourraient se demander si « du fait de son appartenance à tous les savoirs » (Mannoni, 2012 : 6), cette notion ne risque pas d'être considérée comme floue et atomiste. À l'instar de D. Jodelet, nous la définirons comme « une forme de connaissance, socialement élaborée et partagée, ayant une visée pratique et concourant à la construction d'une réalité commune à un ensemble social » (Jodelet, 1998: 36).

Les représentations sociolinguistiques sont « une forme de représentations sociales 》 (Boyer, 2001 : 41), élaborées socialement et partagées par les membres d'une communauté. II s'agit d'un mode spécifique de connaissance, composé d'un ensemble d'informations, opinions et croyances, qui permettent de comprendre comment les individus se représentent les uns les autres à travers leurs langues. Pour le sociologue P. Bourdieu, la langue, le dialecte ou l'accent sont, entre autres, «l'objet de représentations mentales, c'est-à-dire d'actes de perception ou d'appréciation, de connaissance et de reconnaissance, où les agents investissent dans leurs intérêts et leurs présupposés » (Bourdieu, 1980 : 65). À travers nos langues, nous nous construisons une facette de notre identité, celles des autres nous permettent de les catégoriser : il s'agit d'un processus de construction, ou d'un champ de construction permanent de la relation à soi et aux autres.

Pour cerner les représentations relatives aux langues, de soi et de l'Autre, on peut passer par le recueil des discours épilinguistiques au sein d'un groupe, porteurs des images que les locuteurs se font des langues et des pratiques langagières effectives, et qui vont au-delà des perceptions individuelles qui découlent de la conscience propre de chaque individu. En sociolinguistique, l'objectif de ce type de questionnements et d'études est évident, dans la mesure où « les images associées aux langues se présentent comme des témoins de la manière dont sont perçues les situations sociales » (Moreau, 1990 : 407). En ce qui concerne notre corpus, nous faisons l'hypothèse qu'elles jouent un rôle important dans les relations sociales qu'entretiennent les immigrés serbes avec les locuteurs de la langue du pays d'accueil, en opposant de façon nette l'utile à l'agréable, la langue nécessaire à l'intégration à la langue chère au cœur. En partant du principe qu'elles « ne peuvent avoir de sens [...] que dans la mesure où, [...], elles sont « en accord » avec le contexte socioculturel ou politico-social dont elles sont contemporaines » (Mannoni, 2012 : 87), nous proposerons, dans ce qui suit, quelques considérations socio-historiques et linguistiques, afin de situer le groupe observé dans le contexte français actuel, avant de nous pencher sur les représentations sociolinguistiques de ce même groupe. 


\section{Considérations socio-historiques et linguistiques sur la diaspora serbe en France}

Le serbo-croate fait partie des «langues de France » ou du moins des «langues en France $^{2}$ ans le grand recensement intitulé Histoire sociale des langues de France, publié en 2013 par un collectif d'auteurs ${ }^{3}$. Ce recueil a le mérite de réunir les langues minoritaires autochtones, les langues d'outremer ainsi que les langues de l'immigration, ancienne ou récente, sans assise géographique précise. C'est dans cette troisième catégorie qu'il convient de classer le serbo-croate, ou le serbe ${ }^{4}$. Ce type de publications est d'autant plus important qu'on manque de données (ethniques et linguistiques) sur les différentes communautés de diaspora en France. Si les dynamiques migratoires sont bien connues, les statistiques précises pour chaque groupe d'immigrés, à travers l'histoire, restent rares ${ }^{5}$, et les études sociolinguistiques sont, sur ce point, déficitaires.

Traditionnellement terre d'accueil, la France a connu plusieurs vagues d'immigration, surtout depuis la Révolution, lorsqu'elle s'est forgé l'image du pays de la liberté, de la tolérance et des droits de l'homme. Le contexte actuel en France nous montre l'image d'une immigration provenant essentiellement de l'Afrique et du Moyen-Orient, pour des raisons économiques ou géopolitiques, dans un contexte d'hégémonies postcoloniales. Or, à une époque, somme toute pas si lointaine (début ou milieu du XXe siècle), l'immigration était avant tout européenne, et de surcroît davantage choisie que subie (besoin de main-d'œuvre pour industrialiser et/ou reconstruire le pays). Au début du XXe siècle, creusant les inégalités entre les pays riches et les pays pauvres, la révolution industrielle a créé des besoins nouveaux, essentiellement au nord et à l'est du pays. On y accueille des étrangers, souvent en dehors des quartiers résidentiels, pour travailler dans l'industrie lourde et dans les mines.

2. Aux langues de France, outre les langues de la France métropolitaine et des départements et territoires d'outre-mer et les langues non territoriales, pratiquées depuis fort longtemps, comme le rromani ou le judéo-espagnol, ou encore celles arrivées plus récemment, comme l'arabe maghrébin ou le berbère (Kremnitz, 2013 : 36), on peut opposer les langues en France, très nombreuses : toutes les langues de l'immigration parlées sur le territoire français feraient partie de cette dernière catégorie.

3. Le terme qui est utilisé dans cet ouvrage de référence est celui de serbo-croate. Il se justifie encore, dans le domaine linguistique, étant donné l'intercompréhension entre les différentes langues issues du serbo-croate. Sur le plan politique, il convient désormais cependant de parler de serbe, croate, bosnien et monténégrin.

4. Les autres langues de l'immigration qui figurent dans le recueil sont: allemand, anglais, catalan, espagnol, grec, italien, polonais, portugais, roumain, russe, turc, ukrainien, malgache, araméen, persan, tamoul, créole mauricien, créole haïtien, les langues africaines et les langues chinoises. De cette liste sont exclues les langues de France métropolitaine non territoriales, comme l'arabe dialectal, dans toute sa diversité interne, sans doute la principale langue d'immigration sur le sol français. Elles bénéficient d'un traitement à part dans ce recueil collectif.

5. Certes, on peut avoir accès à certaines informations à travers les statistiques publiées par l'INSEE (www.insee.fr), dans la rubrique "étrangers-immigrés », mais celles-ci ne se prêtent pas à une étude sur les immigrés serbes dans la mesure où cette population se trouve dans la rubrique « autres pays d’Europe ». Par ailleurs, en 1999, a été réalisée la grande enquête «Famille» (auprès d'un échantillon représentatif de 380000 personnes), qui a mis en lumière la présence importante d'autres langues que le français dans le cadre familial. En ce qui concerne le serbo-croate, beaucoup de personnes interrogées ont déclaré que leurs deux parents s’adressaient à elles dans cette langue au quotidien durant leur enfance (Héran et al., 2002). 
Une autre vague viendra après la Seconde Guerre mondiale, et s'échelonnera durant les « Trente Glorieuses ». À cette époque, les immigrés qualifiés seront invités à participer notamment au développement de l'industrie automobile, du secteur du bâtiment et des travaux publics. La France signe même un accord de main d'œuvre en 1965 avec la Yougoslavie. Les moins qualifiés travaillent dans des secteurs moins visibles, mais également très demandeurs de main d'œuvre, comme, par exemple, le secteur du textile : "Au XX siècle le quartier du Sentier, à Paris, a vu ainsi se relayer immigrants juifs et arméniens, yougoslaves, puis turcs et pakistanais » (Noiriel, 2002: 19). En effet, les immigrés yougoslaves se fondaient très rapidement dans le paysage socio-économique français de l'après-guerre. La plupart d'entre eux envisageaient de retourner au pays, après la vie active. Généralement bien intégrée, cette population ne se caractérisait pas pour autant par une maîtrise optimale de la langue du pays d'adoption, apprise «sur le tas », dans l'interaction avec leur nouvel environnement social et professionnel. Cependant, la pratique de celle-ci était perçue comme une nécessité, pour leur garantir la sécurité d'emploi, et comme une fierté, preuve de leur capacité d'adaptation. Parallèlement à cette immigration économique, la France a connu également une immigration politique venant de l'ex-Yougoslavie. Les deux populations coexistent ainsi sur le sol français, sans se fréquenter intensément pour autant. Elles seront rejointes par une nouvelle vague d'immigrés, après les guerres de l'ex-Yougoslavie, dans les années 1990, qui ont provoqué le départ d'un grand nombre de jeunes, souvent diplômés, vers les pays occidentaux. Ceux-ci se caractérisent par une véritable volonté d'intégration qui les pousse souvent à rester en dehors du « milieu yougo ». II ne faut pas oublier aussi les réfugiés, sans ressources, qui ont fui les zones de guerre à la même époque.

Ces nouvelles vagues d'immigration ont emporté avec elles des divisions qui avaient contribué à l'éclatement du pays commun, si bien qu'aujourd'hui en France, on a remplacé les associations et les institutions yougoslaves par leurs équivalents serbes, croates, etc. II n'empêche que ces divisions semblent moins visibles à l'étranger, si bien qu'une certaine identité yougoslave ou ex-yougoslave, aussi paradoxale qu'elle puisse paraître plusieurs décennies après l'éclatement du pays, semble exister encore.

De tous les Yougoslaves, les Serbes sont les plus nombreux en France (Bošković, 2006). Les guerres de l'ex-Yougoslavie, et le rôle joué par la Serbie dans celles-ci, ont mis cette population dans une position inconfortable, car la Serbie était souvent stigmatisée dans l'opinion publique française. Ils se sont retrouvés dans un «entre-deux », qui les a parfois poussés jusqu'à afficher des sentiments contradictoires : celui de ne pas faire pleinement partie de la société française en désaccord avec la politique menée par S. Milošević, mais également celui d'être loin de ce qui faisait désormais partie de la «nouvelle » identité serbe : «Certains sont ainsi allés jusqu'à envisager un retour au pays, qui s'est parfois soldé par un échec, leur acculturation française limitant leur pleine réintégration à l'identité serbe » (Colera, $2004: 62$ ). 
Au-delà du cas précis de cette population d'immigrés, la longue coexistence entre les autochtones - à supposer que l'on puisse s'entendre sur la définition de ce terme - et les immigrés, sur le sol français, a fait naître des idées stéréotypées et des représentations plus ou moins figées sur l'autre groupe, qui, comme le veut la définition de ces termes, ne correspondent qu'à une partie de la réalité ou qu'à une partie de la vision que le groupe s'est forgé de cette réalité. Discours xénophobe et stigmatisation de la part de la majorité face au communautarisme de la minorité, ou des immigrés, s'opposent ainsi depuis plusieurs décennies au sein d'une partie de l'opinion publique en France. Dans ce qui suit, nous nous intéresserons précisément au discours sur soi et sur l'Autre, et plus précisément au discours épilinguistique recueilli auprès d'un échantillon de locuteurs issus de l'immigration. Nous présenterons, dans un premier temps, le modèle d'analyse adopté, puis dans un deuxième temps, la constitution du corpus, avant d'analyser les principaux résultats de notre étude de cas.

\section{La méthode d'analyse combinée}

Afin d'analyser les auto- et les hétéro-représentations sociolinguistiques de notre échantillon, nous allons utiliser la méthode d'analyse combinée, développée par Bruno Maurer, avec l'aide de Pierre-Antoine Desrousseaux et Nicolas Serra ${ }^{6}$. Allant au-delà de la théorie structurale d'une représentation sociale, que l'on sait composée, notamment depuis les travaux de J-C. Abric, d'un noyau central et des éléments périphériques (le premier étant stable et résistant au changement, les seconds modulables et perméables aux influences) ${ }^{7}$, elle propose une synthèse entre la démarche quantitative et la démarche qualitative. Selon son auteur, l'un des mérites de cet outil tient au fait qu'il permet de contourner «le caractère trop subjectif des études sociolinguistiques menées par interviews » ainsi que « l'utilisation faite sans précaution de statistiques fantaisistes dans les enquêtes par sondage » (Maurer, 2003 : 1). Sans pour autant rejeter les méthodes existantes, la méthode d'analyse combinée apparaît aujourd'hui comme un outil complémentaire de celles-ci, permettant de travailler avec beaucoup de précision, y compris sur des échantillons réduits.

En ce qui concerne ses différentes étapes, il s'agit dans cette approche, dans un premier temps, de recueillir les éléments de discours relatifs aux langues auprès d'un groupe témoin, qui serviront pour l'élaboration d'un questionnaire de 10, 15 ou 20 items. Les réponses obtenues par l'échantil-

6. La partie linguistique de la méthode a été pensée par Bruno Maurer, professeur en Sciences du Langage à l'Université de Montpellier 3. Les calculs mathématiques et statistiques ont été effectués au préalable par Pierre-Antoine Desrousseaux, enseignant à l'IUT de Béziers, tandis que Nicolas Serra, étudiant à l'IUT de Béziers, était chargé d'automatiser les procédures de traitement aboutissant aux graphes « adhésion-consensus-distance » et aux schémas en quatre couronnes.

7. Voir, par exemple, l'ouvrage dirigé par J-C. Abric, en 1994, qui fait le point sur les avancées théoriques et méthodologiques dans ce champ notionnel, et notamment la structure interne de ces ensembles sociocognitifs que sont les représentations. Ainsi, écrit-il, la détermination du noyau central « est essentiellement sociale, liée aux conditions historiques, sociologiques et idéologiques », tandis que pour le système périphérique, elle est « plus individualisée et contextualisée, beaucoup plus associée aux caractéristiques individuelles et au contexte immédiat et contingent dans lequel sont baignés les individus » (Abric, 1994 : 37). 
Ion choisi (20 personnes minimum) sont ensuite, dans un deuxième temps, saisies dans une feuille de calcul disponible sur le site http://linguiste.iutbeziers.fr/. Celle-ci calcule automatiquement les scores d'adhésion de chaque item, les indices de consensus pour chaque item et les distances entre items inférieures à 1,40 (seuil de significativité retenu pour construire un graphe). Les représentations sociales sont donc analysées à travers trois dimensions, mesurées quantitativement : distance, adhésion et consensus. Les graphes ainsi obtenus sont prêts à être analysés d'un point de vue sociolinguistique, non pas de façon mécanique, mais à la lumière de ce que l'on sait, en tant que chercheurs et sociolinguistes, de la notion de représentation.

L'outil permet de poursuivre l'analyse à travers la création d'un schéma en quatre couronnes, faisant clairement apparaître le noyau central et les éléments périphériques, de façon davantage graduée : la zone de centralité maximum (grande adhésion et grand consensus), la couronne centrale, la périphérie incertaine et la périphérie marginale. On peut y lire le résultat d’une hiérarchisation entre les composantes d'une représentation et l'importance donnée à chaque item que l'on ne peut pas saisir si on travaille sur les pourcentages : « un fort pourcentage de réponses positives (du type : $99 \%$ des gens interrogés pensent que le français vient du latin) n'implique pas pour autant que cette dimension soit qualitativement importante à leurs yeux » (Maurer, 2013 : 20). On peut donc dire que la méthode d'analyse combinée permet de rentrer au cœur d'une représentation sociale des langues.

\section{Recueil du corpus après des locuteurs}

La première étape de notre travail consistait en l'élaboration de questionnaires. Pour cela, nous avons commencé par recueillir les éléments de discours auprès d'un groupe témoin de six personnes, toutes bilingues, en essayant de croiser les différentes variables : deux hommes et quatre femmes, trois enfants d'immigrés et trois expatriés volontaires, une personne née en France et cinq en ex-Yougoslavie, quatre personnes professionnellement intégrées et deux en cours d'intégration. Les membres du groupe témoin ont tous reçu un questionnaire en français, volontairement composé de questions ouvertes, auxquelles ils ont répondu, soit en français, soit en serbe. L'objectif de ce travail leur a été clairement annoncé : étudier quelles représentations les locuteurs d'une langue de l'immigration ont de leur langue et de la langue dominante. Le questionnaire comportait dix questions: 
Tableau 1 : questionnaire groupe témoin.

\begin{tabular}{|c|c|}
\hline \multicolumn{2}{|l|}{ 1. Âge / Sexe: } \\
\hline \multicolumn{2}{|l|}{ 2. Lieu de naissance (pays): } \\
\hline \multicolumn{2}{|c|}{ 3. Depuis quand habitez-vous en France? } \\
\hline \multicolumn{2}{|c|}{ 4. Quelles langues parlez-vous au quotidien? } \\
\hline \multicolumn{2}{|l|}{ 5. Quelles langues écrivez-vous? } \\
\hline \multicolumn{2}{|c|}{ 6. Quelle langue préférez-vous parler ou écrire ... et pourquoi? } \\
\hline \multicolumn{2}{|c|}{ 7. Qu'est-ce qui vous plaît dans les langues que vous parlez? } \\
\hline \multicolumn{2}{|c|}{ 8. Complétez les phrases suivantes: } \\
\hline Le français & Ma langue maternelle \\
\hline C'est une langue qui ... & C'est une langue qui ... \\
\hline C'est une langue que... & C'est une langue que... \\
\hline C'est la langue du ... & C'est la langue du ... \\
\hline C'est la langue de la ... & C'est la langue de la ... \\
\hline Cette langue me fait penser à ... & Cette langue me fait penser à ... \\
\hline \multicolumn{2}{|c|}{ 9. Quelle langue exprime le mieux votre identité ? } \\
\hline \multicolumn{2}{|c|}{$\begin{array}{l}\text { 10. Comment pourriez-vous décrire votre biographie langagière ou votre parcours linguistique? } \\
\text { Comment pourriez-vous décrire votre rapport aux langues (langue francaise et langue maternelle)? }\end{array}$} \\
\hline
\end{tabular}

À partir de leurs réponses, nous avons constitué nos deux questionnaires finaux : l'un sur les représentations du français, l'autre sur les représentations du serbe, que nous avons ensuite soumis à des locuteurs du serbe habitant dans l'Hérault et les Bouches-du-Rhône. La passation des questionnaires ainsi établis a été partiellement facilitée par le fait que certains ont été administrés par personne interposée, laquelle a, elle-même, auparavant répondu aux questions. Chaque personne interrogée a eu à renseigner deux questionnaires de quinze items, sur les auto- et sur les hétéro-représentations. Comme on pourra le voir ci-après, tous les items commencent par «langue qui », «langue de » etc., soit parce que la question a été posée de cette manière, soit parce que nous avons ajouté le mot « langue », là où il était implicite dans le discours de l'enquêté. Nous rappelons que l'élaboration du questionnaire présuppose d'extraire du discours libre les propositions clairement articulées à propos d'un objet de représentation, sans pour autant s'éloigner de ce qui a été dit.

Avec le recul, nous pensons que certaines reformulations auraient été préférables : l'expression « langue utile » peut sembler vague, de même qu'il aurait mieux fallu peut-être remplacer « langue ardue » par «langue difficile». Toujours avec le recul, il nous apparaît que dans le questionnaire serbe pratiquement tous les items sont plutôt positifs. Il aurait fallu peut-être creuser davantage les réponses données par le groupe témoin, ou même élargir celui-ci, afin d’obtenir un questionnaire davantage diversifié. Enfin, dans ce même questionnaire, quatre items expriment de manière diffé- 
rente une idée de passé, de racines, de nostalgie. On pourrait les considérer comme relativement redondants. En effet, les items à forte ressemblance - ce qui tout de même n'est pas le cas ici - peuvent jouer sur les scores finaux. II serait néanmoins intéressant de tester, dans une étape ultérieure du travail, deux questionnaires différents, l'un à dix items, avec un seul exprimant cette idée, l'autre avec cette diversité qui caractérise notre questionnaire actuel, afin de comparer les résultats.

Tableau 2 : Questionnaire « Représentations du français par un groupe d’immigrés de langue maternelle serbe »

\begin{tabular}{|c|c|c|}
\hline \multicolumn{3}{|c|}{ Pour moi, le français c'est une / c'est la ... } \\
\hline $\mathbf{n}^{\circ}$ & Propositions & $\begin{array}{c}\text { Notes } \\
(+2,-2,+1,-1,0)\end{array}$ \\
\hline 1. & langue utile & \\
\hline 2. & langue de la mode & \\
\hline 3. & langue importante sur le plan national & \\
\hline 4. & langue qu'il faut bien articuler & \\
\hline 5. & langue que je parle quand je parle sérieusement & \\
\hline 6. & langue qui fait partie de mon quotidien & \\
\hline 7. & langue de l'intégration & \\
\hline 8. & langue que je parle avec un accent & \\
\hline 9. & langue de Victor Hugo et de grands auteurs & \\
\hline 10. & langue qui permet d'exprimer une multitude de nuances & \\
\hline 11. & langue de mon « autre moi » & \\
\hline 12. & langue qui me fait penser à ce que je n'aime pas dans ce pays & \\
\hline 13. & langue que j'associe à l'âge adulte & \\
\hline 14. & langue dont je maîtrise l'orthographe correctement & \\
\hline 15. & langue ardue & \\
\hline
\end{tabular}


Tableau 3 : «Représentations du serbe par un groupe d'immigrés de langue maternelle serbe »

\begin{tabular}{|c|c|c|}
\hline \multicolumn{2}{|c|}{ Pour moi, le serbe c'est une / c'est la ... } & Notes \\
\hline$n^{\circ}$ & Propositions & $(+\mathbf{2}, \mathbf{- 2}, \mathbf{+ 1 , - 1 , 0 )}$ \\
\hline 1. & langue qui m'évoque de beaux paysages & \\
\hline 2. & langue de mon identité & \\
\hline 3. & langue qui rend ma voix plus belle et plus naturelle & \\
\hline 4. & langue qui me fait penser aux vacances d'été & \\
\hline 5. & langue d'Ivo Andric et de grands auteurs & \\
\hline 6. & langue des plaisanteries & \\
\hline 7. & langue de mes grands-parents & \\
\hline 8. & langue du respect & \\
\hline 9. & langue de l'amitié & \\
\hline 10. & langue qui permet de tout dire & \\
\hline 11. & langue de mes racines & \\
\hline 12. & langue des premières années de mon enfance & \\
\hline 13. & langue qui m'évoque les bons souvenirs du passé & \\
\hline 14. &
\end{tabular}

Les répondants étaient invités à noter par +2 les trois propositions avec lesquelles ils étaient le plus d'accord, par +1 les trois propositions avec lesquelles ils étaient assez d'accord, par -2 les trois propositions avec lesquelles ils n'étaient pas du tout d'accord, par -1 les trois propositions avec lesquelles ils n'étaient pas d'accord et par 0 les trois propositions restantes.

\section{Interprétation des résultats}

Nous avons pu collecter quarante questionnaires valides ${ }^{8}$. Il s'agit, certes, d'un échantillon modeste qui se prête difficilement à une grande extrapolation. Néanmoins, avec la méthode d'analyse combinée, un tel corpus se prête à une multitude d'observations intéressantes : on peut ainsi calculer le consensus au sein d'un groupe d'immigrés serbes à l'égard des éléments de leurs auto- ou hétéro-représentations sociolinguistiques ou encore les scores d'adhésion ou de rejet par rapport aux items qui leur ont été proposés. En plus des graphes, cette méthode offre la possibilité de construire des schémas en couronnes, permettant de répartir les items entre éléments centraux et éléments périphériques, et de montrer les zones significatives de chaque représentation. Il va de soi que les

8. Nous avons écarté de notre échantillon les questionnaires incomplets (ratures, oublis, mauvaise compréhension de consignes), et ceux dans lesquels les répondants ont indiqué que leur langue maternelle n'était pas le serbe, mais le croate, le bosnien ou le monténégrin. Une fois l'échantillon complété, ces derniers pourraient faire l'objet d'un traitement différencié, utilisable dans une optique comparative. 
éléments d'analyse et d'interprétation que nous proposons ne concernent que le corpus recueilli et n'ont aucune valeur de généralisation autre qu'heuristique.

Notre travail ici se concentrera seulement sur deux dimensions de la méthode d'analyse combinée - l'adhésion et le consensus -, sans aborder la troisième - la distance. B. Maurer souligne d'ailleurs la fragilité « de l'hypothèse reliant densité (faibles distances entre items) et centralité », les deux premières dimensions étant plus révélatrices pour lui « du comportement du groupe à l'égard de la représentation » (Maurer, 2013:47;1). Par ailleurs, les feuilles de calcul pour nos deux questionnaires n'indiquent aucune distance significative entre items (inférieure à la valeur 1,40).

\subsection{Représentation de la langue française}

Voici, dans un premier temps, le graphe « adhésion-consensus » concernant la représentation de la langue française :

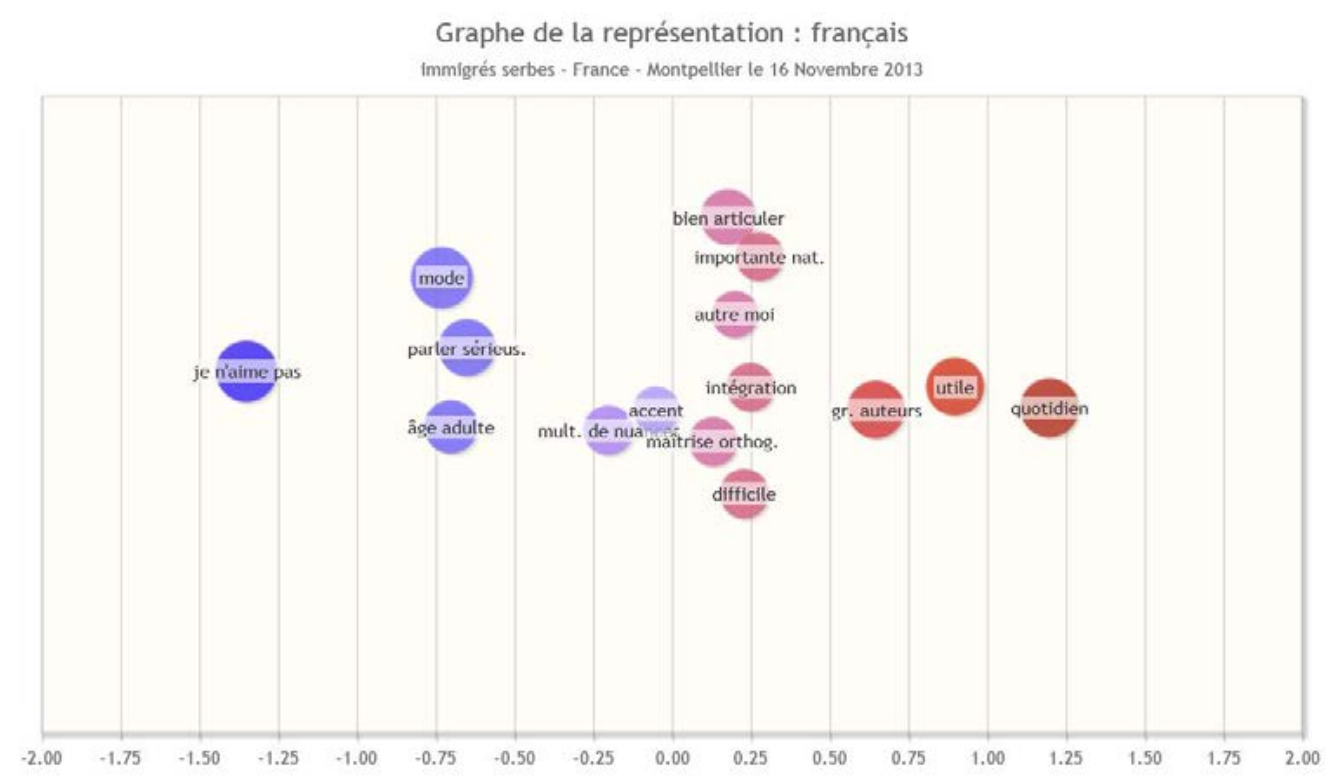

Graphe 1 : langue française.

L'adhésion se lit sur la ligne horizontale du graphe. À droite du schéma se trouvent les éléments qui obtiennent la plus grande adhésion, à gauche, c'est le rejet qui domine; les couleurs allant du bleu au rouge permettent facilement de situer les deux polarités d'un continuum. La langue qui fait partie de mon quotidien et la langue utile s'opposent à la langue qui me fait penser à ce que je n'aime pas dans ce pays. On voit que les répondants choisissent de rejeter massivement l'idée que le français pourrait renvoyer à quelque chose de négatif, et lui attribuent une valeur essentiellement utilitaire, dans la vie au quotidien. L'un des principaux stéréotypes concernant la langue française (langue de la mode), souvent véhiculé par les médias, est également rejeté par les immigrés, leurs préoccupations étant souvent loin de ce secteur d'activité. 
Le consensus se mesure par la taille des cercles du graphe. On peut constater qu'il est important pour un grand nombre d'items, parmi lesquels : langue qui me fait penser à ce que je n'aime pas dans ce pays (l'item qui obtient le plus grand indice de consensus), langue de la mode, langue qui fait partie de mon quotidien, langue utile, langue de Victor Hugo et de grands auteurs, langue que je parle quand je parle sérieusement. Les quatre items qui ont provoqué soit la plus grande adhésion, soit le plus grand rejet, génèrent également le plus grand consensus au sein du groupe. En revanche, le consensus est minimal pour les items suivants : langue de mon « autre moi » et langue que je parle avec un accent. On pourrait peut-être voir ici un certain paradoxe induit par la dispersion ou par le désaccord des sujets dans les réponses : certains immigrés interrogés semblent assumer leur position d'étrangers (le français reste une langue étrangère qui ne participe pas à la construction de leur identité), tandis qu'on peut observer chez d'autres une volonté de montrer leur intégration dans la société française, à travers une bonne compétence linguistique et l'absence d'un accent - ce dernier facteur pouvant être très stigmatisant.

Dans la mesure où il n'est pas toujours aisé de mesurer à l'œil nu le positionnement exact d'un item, ni de juger de la taille du cercle, la feuille de calcul permet de chiffrer les données de façon précise:

Tableau 4 : Feuille de calcul (langue française)

\begin{tabular}{|c|c|c|l|c|c|c|c|c|}
\hline $\begin{array}{l}\text { Étude de la langue: français -- Population: immigrés serbes -- Pays: France -- Ville: Montpellier -- } \\
\text { Date: 16 novembre 2016 }\end{array}$ & Utile & Mode & $\begin{array}{c}\text { I m p o r - } \\
\text { t a n t e } \\
\text { nat. }\end{array}$ & $\begin{array}{c}\text { Bien } \\
\text { articuler }\end{array}$ & $\begin{array}{c}\text { Parler } \\
\text { sérieus. }\end{array}$ & $\begin{array}{c}\text { Quoti- } \\
\text { dien }\end{array}$ & $\begin{array}{c}\text { Intégra- } \\
\text { tion }\end{array}$ & Accent \\
\hline Score d'adhésion & 0,9 & $-0,73$ & 0,28 & 0,18 & $-0,65$ & 1,2 & 0,25 & $-0,05$ \\
\hline Indice de consensus & 0,082 & 0,087 & 0,069 & 0,08 & 0,081 & 0,084 & 0,069 & 0,066 \\
\hline
\end{tabular}

\begin{tabular}{|c|c|c|c|c|c|c|c|}
\hline $\begin{array}{l}\text { Étude de la langu } \\
\text { Date: } 16 \text { novembre } 20\end{array}$ & : français & Popula & : immigrés & serbes -- & ays: France & -- Ville: & tpellier \\
\hline & Gr. auteur & $\begin{array}{l}\text { Mult. de } \\
\text { nuances }\end{array}$ & Autre moi & $\begin{array}{l}\text { Je n'aime } \\
\text { pas }\end{array}$ & Âge adulte & $\begin{array}{l}\text { Maîtrise } \\
\text { orthog. }\end{array}$ & Difficile \\
\hline Score d'adhésion & 0,65 & $-0,2$ & 0,2 & $-1,35$ & $-0,7$ & 0,13 & 0,23 \\
\hline Indice de consensus & 0,082 & 0,071 & 0,066 & 0,088 & 0,076 & 0,07 & 0,07 \\
\hline
\end{tabular}

Le schéma en couronnes, pour la langue française, est organisé en quatre cercles : la zone de centralité maximum (langue qui fait partie de mon quotidien), la couronne centrale (sept items, dont langue utile et langue de la mode), la périphérie incertaine (six items, dont langue importante sur le plan national et langue de l'intégration) et la périphérie marginale (langue qui me fait penser à ce que je n'aime pas dans ce pays). 


\section{Schéma en couronnes de la structure de la représentation sociale}

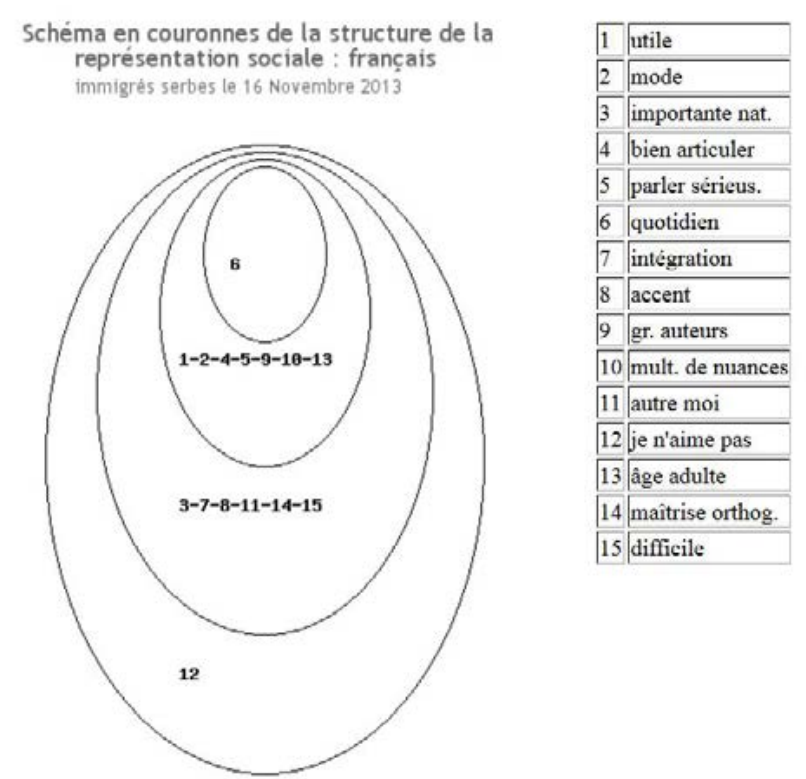

Schéma 1 : Langue française

On voit que dans ce schéma s'opposent fortement un item qui forme, seul, la zone de centralité maximum (langue qui fait partie de mon quotidien), et un item - un seul également - fortement repoussé à la marge (langue qui me fait penser à ce que je n'aime pas dans ce pays). L'item qui se trouve dans la zone de centralité maximum est celui qui détermine la signification de la représentation. C'est l'élément le plus stable et le plus pérenne de la représentation. Nous avons vu dans le graphe «adhésion-consensus » qu'il a obtenu également la plus grande adhésion du groupe et un consensus relativement important. L'item qui se trouve dans la périphérie marginale est celui qui est peut-être le plus près du contexte, dont il dépend. Il a généré le plus grand rejet, mais également le plus grand consensus au sein du groupe interrogé. 


\subsection{Représentation de la langue serbe}

Observons, à présent, le graphe « adhésion-consensus » concernant la représentation de la langue serbe $^{9}$ :

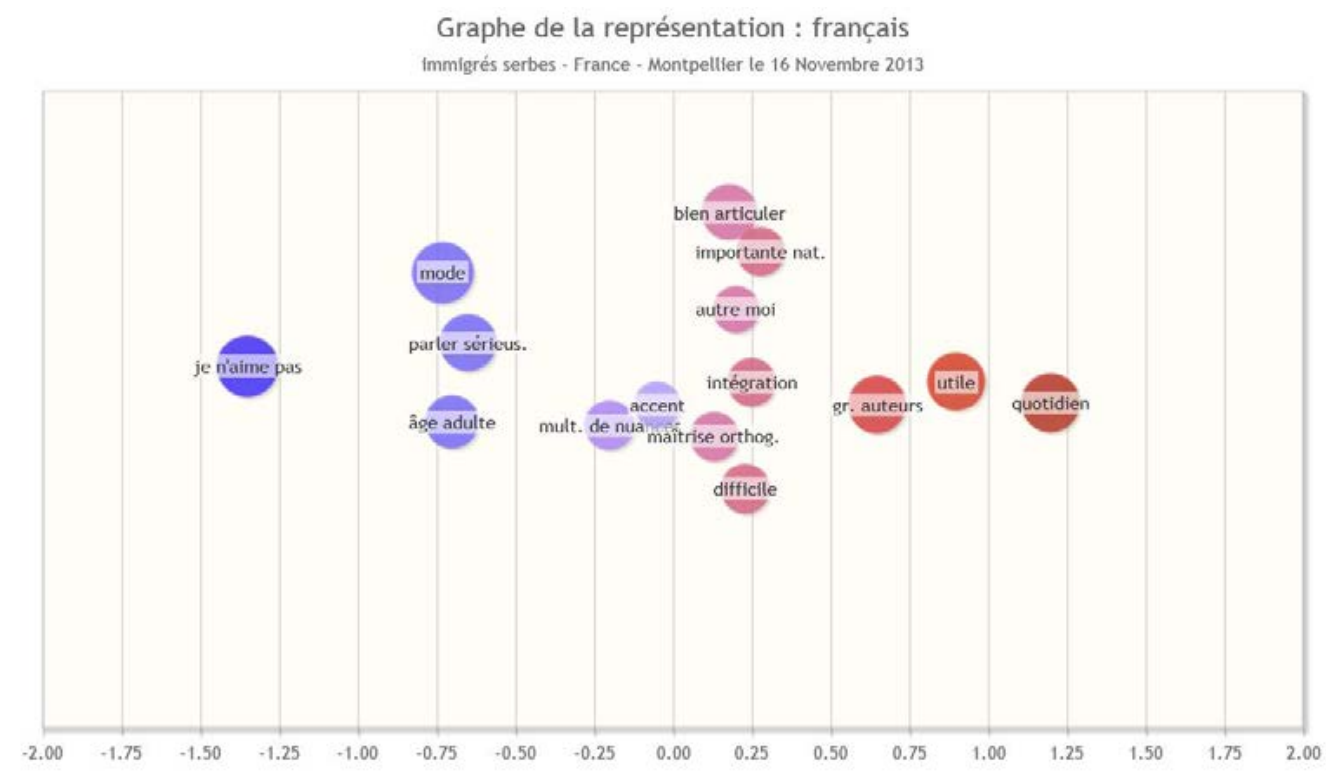

Graphe 2: Langue serbe

Ici aussi, le graphe nous permet de voir de façon claire le système de valeurs dans lequel la langue maternelle est insérée. L'adhésion nous indique l'importance aux yeux des personnes interrogées des différents éléments qui participent à la construction de la représentation, les éléments les plus importants pour le groupe étant situés à droite. On voit qu'environ la moitié des items est évaluée positivement (8 items), l'autre moitié négativement (7 items). Le résultat du calcul du score moyen attribué par le groupe se lit comme suit : langue de mon identité et langue de mes racines (la plus grande adhésion) face à langue qui rend ma voix plus belle et plus naturelle et langue du respect (le plus grand rejet). Le rejet pourrait peut-être s'expliquer par la formulation des deux items proposés. Sortis de leur contexte, ils apparaissent quelque peu abstraits. Il nous semble intéressant de remarquer que le rejet concerne également l'item 6 (langue d'Ivo Andric et de grands auteurs). II est, certes, faible, mais on reste quand même dans la polarité négative du graphe. On peut peut-être faire l'hypothèse que ce résultat est proportionnel à la catégorie socio-économique dominante dans l'échantillon : une population davantage ouvrière qu'intellectuelle. Par ailleurs, certains de ces immigrés (notamment ceux nés en France) n'ont pas suivi une scolarité en serbe, leur connaissance de la langue et des éléments culturels (fût-ce le prix Nobel de la littérature) ne va pas au-delà du

9. Dans la légende du graphe, on peut lire le « serbo-croate » et non pas le « serbe ». Cela est dû simplement au nom que nous avons donné à notre saisie, le 16 novembre 2013. 
niveau superficiel. Cela montre également, si besoin était, à quel point l'école joue un rôle dans la structuration de nos représentations.

Le tableau suivant permet de lire les scores d'adhésion calculés automatiquement:

Tableau 5 : Feuille de calcul (langue serbe)

\begin{tabular}{|c|c|c|c|c|c|c|c|}
\hline & Complexe & $\begin{array}{c}\text { Beaux } \\
\text { paysages }\end{array}$ & Identité & Belle voix & Vacances & $\begin{array}{c}\text { Gr. } \\
\text { auteurs }\end{array}$ & $\begin{array}{c}\text { Plaisante- } \\
\text { ries }\end{array}$ \\
\hline Score d'adhésion & $-0,43$ & $-0,6$ & 0,9 & $-0,78$ & 0,1 & 0,18 & 0,2 \\
\hline Indice de consensus & 0,072 & 0,069 & 0,07 & 0,081 & 0,067 & 0,074 & 0,073 \\
\hline
\end{tabular}

\begin{tabular}{|c|c|c|c|c|c|c|c|c|}
\hline \multicolumn{9}{|c|}{$\begin{array}{l}\text { Étude de la langue: serbo-croate -- Population: immigrés serbes -- Pays: France -- Ville: Montpellier -- } \\
\text { Date: } 16 \text { novembre } 2016\end{array}$} \\
\hline & $\begin{array}{l}\text { Grands- } \\
\text { parents }\end{array}$ & Respects & Amitié & Tout dire & Racines & Enfance & $\begin{array}{c}\text { Sonori- } \\
\text { tés } \\
\text { franc. }\end{array}$ & $\begin{array}{l}\text { Souve- } \\
\text { nirs }\end{array}$ \\
\hline Score d'adhésion & 0,2 & $-0,65$ & $-0,23$ & 0,08 & 0,73 & 0,43 & 0,43 & 0,2 \\
\hline Indice de consensus & 0,075 & 0,081 & 0,08 & 0,066 & 0,073 & 0,07 & 0,067 & 0,067 \\
\hline
\end{tabular}

Le même tableau fait apparaître les résultats pour l'indice de consensus, qui indique la position du groupe à l'égard des éléments constitutifs de la représentation. Il est maximal pour les items suivants : langue qui rend ma voix plus belle et plus naturelle, langue du respect, langue de l'amitié, langue de mes grands-parents, langue d'Ivo Andric et de grands auteurs. On voit clairement sur quels éléments la représentation s'ancre dans la réalité, la taille des cercles étant proportionnelle à la force du consensus, celui-ci étant le résultat des positions individuelles identiques. Deux de ces éléments sont déjà fortement évalués en ce qui concerne le rejet, tandis que deux items fortement marqués en termes d'adhésion n'obtiennent pas un grand indice de consensus.

Pour la langue maternelle, le schéma en couronne est organisé autour de deux cercles, la zone de centralité maximum et celle de la périphérie marginale ne comportant aucun élément. Dans la couronne centrale se trouvent huit items, parmi lesquels langue complexe et langue qui rend ma voix plus belle et plus naturelle en français, et dans la périphérie incertaine, sept items de moindre importance dans la représentation, dont langue qui m’évoque de beaux paysages et langue de mon identité. La plupart des items constituant la zone de la couronne centrale sont fortement consensuels, tandis qu'on peut observer l'absence de consensus dans la zone de la périphérie incertaine. 


\section{Schéma en couronnes de la structure de la représentation sociale}

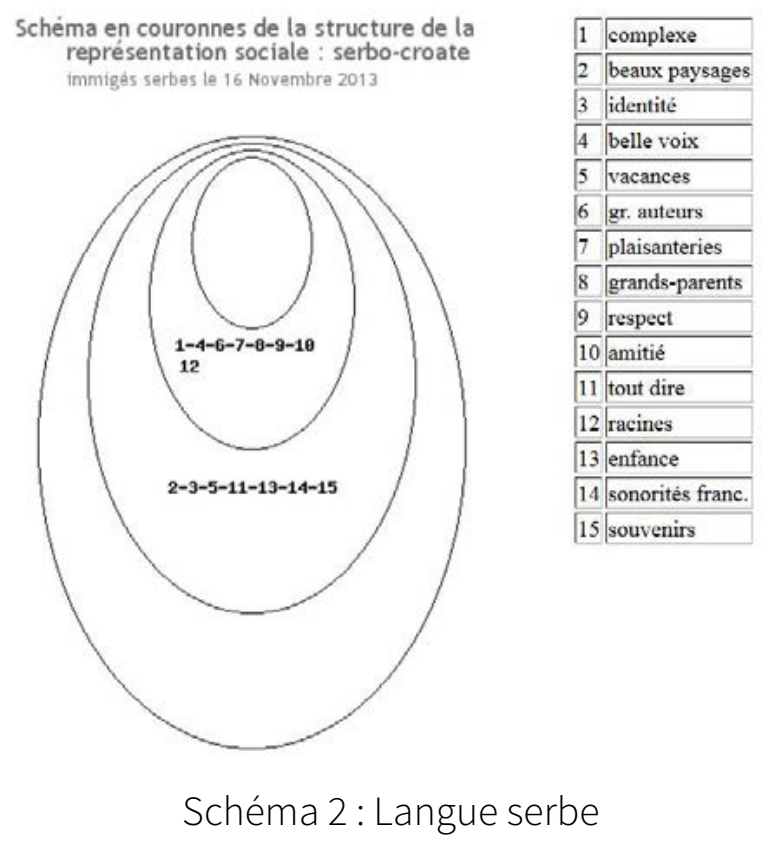

Telle qu'elle est présentée dans le schéma en couronnes, la représentation de la langue serbe apparaît comme une représentation faiblement structurée. On sait que les items affectés par les indices de consensus importants se situent généralement dans les deux zones qui, ici, ne sont pas sollicitées (centralité maximum et périphérie incertaine). Au final, elle est constituée de deux ensembles relativement équilibrés en nombre, se rattachant pour la plupart au registre de l'affectivité.

\subsection{Regard croisé}

Après avoir décrit chacune des représentations dans le groupe donné, nous pouvons proposer quelques éléments de comparaison. De façon générale, le graphe est moins polarisé pour le serbe, et le schéma en couronnes est plus simple. Du point de vue de l'organisation interne de cette figure, on constate que la représentation du français est organisée autour de deux éléments, centre et périphérie, chacun d'eux se divisant en deux ensembles, tandis que la représentation du serbe se limite en deux éléments non différenciés.

L'hypothèse avancée dans l'introduction semble se confirmer : les répondants opposent de façon nette la langue nécessaire à l'intégration à la langue chère au cœur, en adhérant majoritairement à la langue qui fait partie de mon quotidien et à la langue utile pour le français face à la langue de mon identité et à la langue de mes racines pour le serbe. La représentation du français est mieux structurée que celle du serbe, l'adhésion aux items choisis est plus forte (1,2 - pour la langue qui fait partie de mon quotidien face à la langue de mon identité - 0,9), le rejet plus prononcé (-1,35 - pour la 
langue qui me fait penser à ce que je n'aime pas dans ce pays face à la langue qui rend ma voix plus belle et plus naturelle en français - 0,78 ). La répartition entre la polarité positive et la polarité négative est semblable, avec une légère préférence dans le graphe concernant la représentation du français pour les items évalués positivement ( 9 contre 8 pour le serbe). Les éléments pragmatiques semblent primer dans le cas de la langue du pays d'accueil, son importance au quotidien et sur le plan de l'intégration et son utilité sont mis au premier plan. La nostalgie, les souvenirs des bons moments et l'affectivité dominent dans le cas de la langue maternelle. Dans les deux cas, il ne faut pas perdre de vue le contexte dans lequel cette étude de cas a été réalisée, et le déséquilibre fonctionnel qui caractérise cette situation de contact.

\section{Conclusion}

L'idée de départ de ce travail était d'apporter des éléments sur la façon dont sont perçues les langues serbe (langue maternelle des répondants qui constituent notre échantillon) et française (langue de leur pays d'accueil), en utilisant comme grille de lecture et d'analyse, la méthode d'analyse combinée. Les résultats présentés dans ce travail ont avant tout un caractère original - il s'agit, à notre connaissance, de la première étude de ce genre en contexte français. Ils sont aussi porteurs de sens, dans la mesure où les individus se définissent aussi par rapport à la langue qu'ils parlent et aux langues qui font partie de leur identité, souvent composite et à multiples facettes.

Dans une étape ultérieure, l'apport de notre étude de cas pour un projet sur les représentations des langues et des identités en Méditerranée pourrait consister, entre autres, dans l'observation que l'on pourra faire de la manière dont la diversité interne d'un ensemble de langues proches linguistiquement (serbe, croate, bosnien et monténégrin) se confronte, dans le discours, à la question de l'unité de l'identité française, tout aussi complexe ou paradoxale. 


\section{Références}

Abric, Jean-Claude (dir.) (1994), Pratiques sociales et représentations, Paris, Presses universitaires de France.

Bošković, Sanja (2006), "L'émigration yougoslave à Paris : entre intégration et exclusion », Les cahiers du MIMMOC, n 1, disponible sur http://mimmoc.revues.org/167. [Page consultée le 29 juin 2016.]

Bourdieu, Pierre (1980), «L'identité et la représentation : éléments pour une réflexion critique sur l’idée de région », Actes de la recherche en sciences sociales, vol. 35, p. 63-72.

Boyer, Henri (2001), Introduction à la sociolinguistique, Paris, Dunod.

Colera, Christophe (2004), «Tensions franco-yougoslaves et constructions identitaires des Serbes en France », Hommes et migrations, n 1250 , p. 62-75.

Héran, François, Alexandra Filhon et Christine Deprez (2002), « La dynamique des langues en France au fil du XXe siècle », Population \& sociétés, n 376, disponible sur https://www.ined.fr/fichier/s rubrique/18724/pop_et_soc_francais_376.fr.pdf. [Page consultée le 29 juin 2016.]

Jodelet, Denise (1989), Les représentations sociales, Paris, Presses universitaires de France.

Kremnitz, Georg (dir.) (2013), Histoire sociale des langues de France, Rennes, Presses universitaires de Rennes.

Mannoni, Pierre (2012), Les représentations sociales, $6^{e}$ éd., Paris, Presses universitaires de France. [1'éd., 1998].

Maurer, Bruno (2013), Représentations sociales des langues en situation multilingue : la méthode d'analyse combinée, nouvel outil d'enquête, Paris, Éditions des archives contemporaines.

Moreau, Marie-Louise (1990), "Des pilules et des langues : le volet subjectif d'une situation de multilinguisme au Sénégal », dans Elhousseine Gouaini et Ndiassé Thiam (dir.), Des langues et des villes, Paris, Didier Érudition, p. 407-420.

Noiriel, Gérard (2002), Atlas de l'immigration en France, Paris, Éditions Autrement. 